\title{
Chip-based optical microscopy for imaging membrane sieve plates of liver scavenger cells
}

Øystein Helle, Cristina Øie, Peter McCourt, Balpreet Ahluwalia

Øystein Ivar Helle, Cristina lonica Øie, Peter McCourt, Balpreet Singh Ahluwalia, "Chip-based optical microscopy for imaging membrane sieve plates of liver scavenger cells," Proc. SPIE 9554, Nanoimaging and Nanospectroscopy III, 955400 (26 August 2015); doi: 10.1117/12.2187277

EPIE SPIE Nanoscience + Engineering, 2015, San Diego, California, United States 


\title{
Chip-based optical microscopy for imaging membrane sieve plates of liver scavenger cells
}

\author{
Øystein Ivar Helle ${ }^{1}$, Cristina Ionica $\varnothing_{i e^{2}}$, Peter McCourt $^{2}$, Balpreet Singh Ahluwalia* \\ UiT-The Arctic University of Norway \\ Department of Physics and Technology ${ }^{1}$, Department of Medical Biology ${ }^{2}$ \\ 9037 TROMSØ, NORWAY \\ E-mail" : Balpreet.singh.ahluwalia@uit.no
}

\begin{abstract}
The evanescent field on top of optical waveguides is used to image membrane network and sieve-plates of liver endothelial cells. In waveguide excitation, the evanescent field is dominant only near the surface $(\sim 100-150 \mathrm{~nm})$ providing a default optical sectioning by illuminating fluorophores in close proximity to the surface and thus benefiting higher signal-to-noise ratio. The sieve plates of liver sinusoidal endothelial cells are present on the cell membrane, thus near-field waveguide chip-based microscopy configuration is preferred over epi-fluorescence. The waveguide chip is compatible with optical fiber components allowing easy multiplexing to different wavelengths. In this paper, we will discuss the challenges and opportunities provided by integrated optical microscopy for imaging cell membranes.
\end{abstract}

\section{INTRODUCTION TO WAVEGUIDE CHIP-BASED MICROSCOPY}

Fluorescence microscopy has emerged as a vital tool in modern bio-medical imaging and diagnosis. Different fluorescent stains (dyes) that bind specifically to sub-cellular organelles, membrane, proteins, allow optical microscopy to provide specific intra-cellular level information. In total internal reflection fluorescence microscopy (TIRF), the surface evanescent field is used to illuminate the sample [1-5]. The evanescent field decays exponentially above the surface and is used to illuminate thin section of the sample (typically 150-200 nm). Unlike epi-fluorescence where the entire sample is illuminated, TIRF microscopy illuminates only a thin section of the cell providing a high signal-to-noise ratio by reducing the background signal and low photo-toxicity. TIRF microscopy is a preferred methodology for imaging targets or biological phenomena in close proximity to the cell membrane, such as cell membrane trafficking, cell adhesion points or structures present on cell membrane.

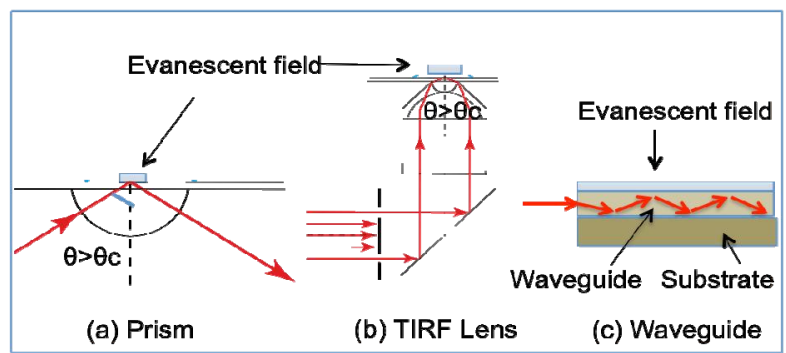

Figure 1: Different techniques to generate an evanescent field. a) Prism with angle of incidence greater than the critical angle, $\theta>\theta c$. b) TIRF objective. c) Evanescent field on top of a waveguide.

Different techniques can be used to generate the evanescent field as shown in Fig. 1. The most commonly used method is a total internal reflection (TIR) objective lens. The TIR lens is used to generate the evanescent field at the surface of the cover slip, and illuminates the samples residing directly on top of cover slips. As shown in Fig. 1c, the light can be confined inside an optical waveguide and guided along a pre-fabricated pattern. On the surface of a waveguide, an evanescent field is present along the entire length of the waveguide (Fig 3c). The surface evanescent field of a waveguide can also be used for TIRF microscopy, in the same way as a TIR objective lens (Fig 1) [3-5]. In

Nanoimaging and Nanospectroscopy III, edited by Prabhat Verma, Alexander Egner, Proc. of SPIE

Vol. 9554, 95540O · C 2015 SPIE · CCC code: 0277-786X/15/\$18 · doi: 10.1117/12.2187277 
conventional TIR objective lens based microscopy both the illumination and the collection light path are guided thorough the TIR objective lens. Here, a waveguide-chip provides the illumination to the sample and any standard objective lens can be used to collect the signal, thus decoupling the illumination and the collection light path.

\section{LIVER SINUSOIDAL ENDOTHELIAL CELLS}

The liver cells such as Kupffer cells (KCs) and liver sinusoidal endothelial cells (LSECs) are engaged in blood clearance activity [6-9]. The LSECs, which line the very small blood vessels (sinusoids) of the liver, possess unique morphological characteristics called "fenestrations" (small holes grouped in sieve plates) with a mean diameter of approximately 100 $\mathrm{nm}$. Together these cell types make up the body's most powerful scavenger system. In mammals, the KCs directly engulf and remove larger targets $(>200 \mathrm{~nm})$ whereas LSECs eliminate smaller targets $(<200 \mathrm{~nm})$ via clathrin mediated endocytosis. LSECs are uniquely characterized structurally with fenestrations (nano-holes of $\sim 50-200 \mathrm{~nm}$ diameter) grouped in sieve plates (Figure 2), which allow small soluble material, but not larger particles to pass across the sinusoidal wall to the underlying parenchymal cells. As fenestrations and sieve plates are present only on the cell membrane, TIRF (total internal reflection microscopy) configuration is preferred over epi-fluorescence.

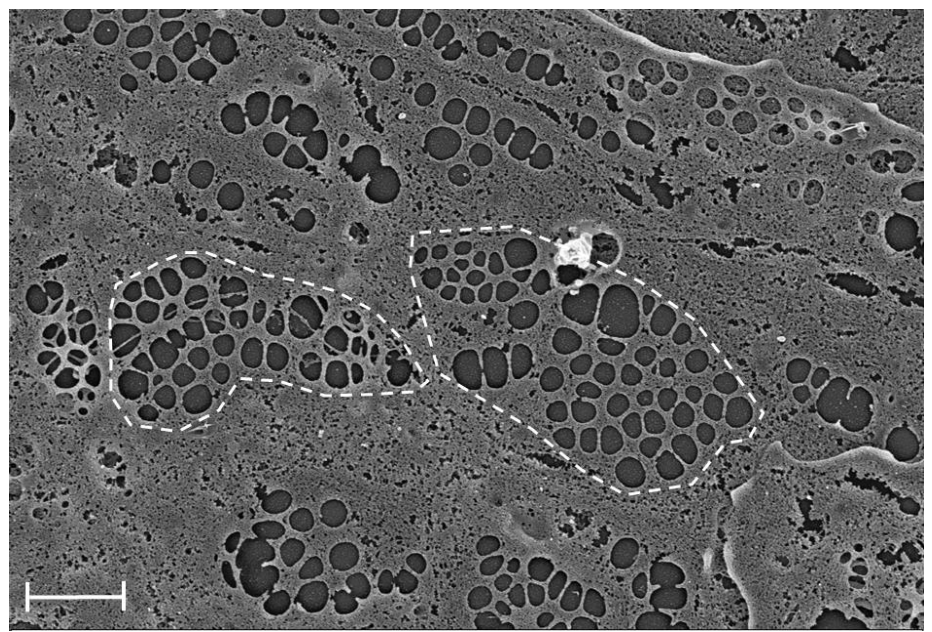

Figure 2. Scanning electron micrograph of rat LSEC plasma membrane showing fenestrations clustered in sieve plates (dotted line). Scale bar $=1 \mu \mathrm{m}$.

\section{METHODOLOGY}

Waveguide material: Low loss, low auto-fluorescence optical waveguides for visible light $(532 \mathrm{~nm})$ are required for waveguide-chip based microscopy. Waveguides made of a material with a high refractive index, e.g. tantalum pentoxide $\left(\mathrm{Ta}_{2} \mathrm{O}_{5}\right)$ or silicon nitride $\left(\mathrm{Si}_{3} \mathrm{~N}_{4}\right)$, give high intensity in the evanescent field and a smaller penetration depth. In this work we used optical waveguides made of tantalum pentoxide $\left(\mathrm{Ta}_{2} \mathrm{O}_{5}\right), \mathrm{n}=2.1$ and high refractive index contract $(\Delta \mathrm{n})$ of 0.75 when compared with $\mathrm{SiO}_{2}(\mathrm{n}=1.45)$. We have previously optimized the fabrication process for $\mathrm{Ta}_{2} \mathrm{O}_{5}$ waveguides [1011] and have used them for waveguide trapping application [12-15]. Strip waveguides with a strip height of $110 \mathrm{~nm}$ on silcon dioxide substrates were used for imaging. A $5 \mu \mathrm{m}$ thick $\mathrm{SiO}_{2}$ was present below the waveguide layer as a cladding layer. Due to the large size of the imaged cells, the width of the waveguides used are either $50 \mu \mathrm{m}$ or $100 \mu \mathrm{m}$. This ensures that the imaging region will be suited for the rathter large stretch of the LSECs.

Cell isolation staining: Rat LSECs were prepared by collagenase perfusion of the liver, low speed differential centrifugation and Percoll gradient sedimentation [16], followed by KCs depletion by seeding the nonparenchymal fraction onto plastic culture dishes. This resulted in a cell suspension enriched in LSECs. Human LSECs were also isolated from patients undergoing liver resections based on immunomagnetic selection. The cells were seeded on waveguides pre-coated with fibronectin and allowed attachment for $1 \mathrm{~h}$. Non attached cells and debris were removed, and after another $1 \mathrm{~h}$ of incubation, the cell were fixed with $4 \%$ PFA for 10 minutes at RT. Following a 30 min incubation with $1 \%$ BSA to minimize unspecific binding of the dyes, the cells were stained for actin filaments by incubating the 
cells for 20 min at $37^{\circ} \mathrm{C}$ with Alexa Fluor 647 Phalloidin (1:40 dilution in PBS). After extensive washes with PBS, the plasma membrane was stained by incubating the cells for 10 min at RT with Cell Mask Orange (working concentration of $1.25 \mathrm{ng} / \mathrm{ml}$ in PBS).

Experimental set-up: The experimental set-up employed is shown in Figure 3. The two lasers beams $532 \mathrm{~nm}$ and 638 $\mathrm{nm}$ were used for TIRF excitation and were combined and coupled into the waveguides using an 50X 0.5 NA objective lens. The input coupling objective lens was kept on a translation stage to optimize the coupling efficiency on to the waveguide chip. An upright microscope (Olympus) with adequate emission filter sets was used, also this mounted on a translation stage allowing freedom to move the field of view around on the waveguide chip. The images were captured using a sensitive sCMOS camera (Hamamatsu Orca Flash 4). For epi-fluorescence imaging, another $532 \mathrm{~nm}$ laser was introduced from the top of the microscope as shown in Fig. 3. The sample chamber was made using thin PDMS layer (110 nm thickness), with an opening at the center to introduce the cells and image buffer. The thickness of the PDMS chamber was matched to the shortest working distance of the objective lenses used for imaging, to ensure the best possible image quality. The PDMS opening was sealed by a cover slip and different N.A. objective lens were used to acquire images. The cells were placed on top of the waveguide and were illuminated by the evanescent field of the waveguide for 2D imaging and by the propagating laser beam for epi-fluorescence mode.

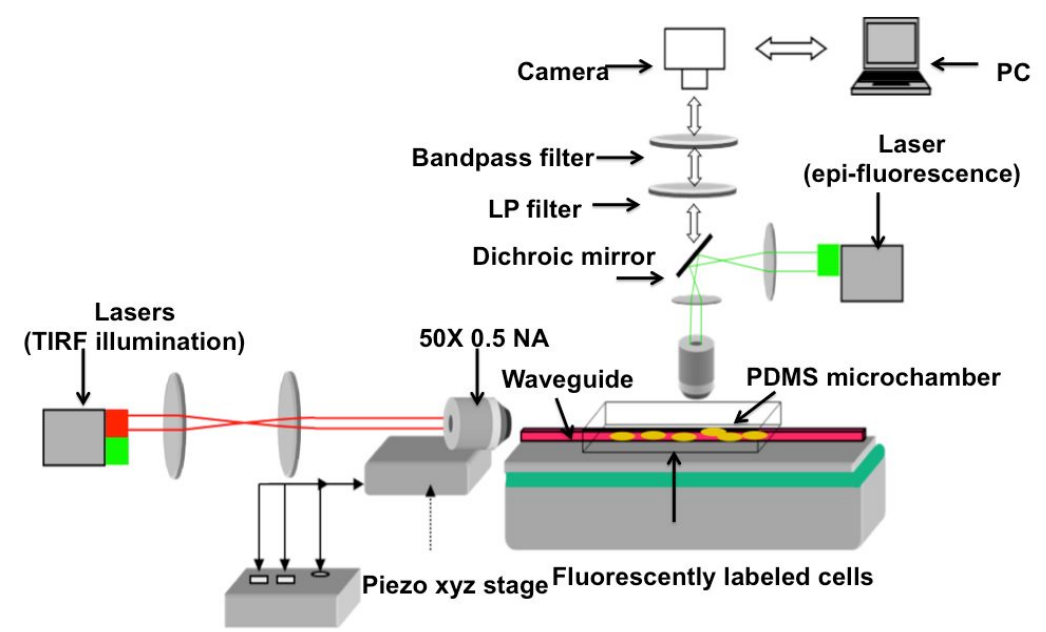

Figure 3 Experimental set-up of waveguide-chip based microscopy. Lasers (532 nm/638 nm) were used for 2D chip-based imaging and another $532 \mathrm{~nm}$ laser was used for epi-fluorescence from the top of an upright microscope.

\section{WAVEGUIDE CHIP-BASED IMAGING OF LIVER CELLS}

The primary LSECs were immobilized directly on top of waveguides, fixed within 3 hours after the isolation and stained. The waveguide is coated with a thin layer of fibronectin to help immobilization of LSECs on the waveguide surface. Figure 4a shows a bright field image of LSECs and Figure 3c shows the cells imaged using the evanescent field of the waveguide. The cell membrane network and sieve-plates (dark areas in the membrane) are imaged, while Fig. 3b shows an epi-fluorescence image of the same cell. In epi-fluorescence excitation the cell nucleus become emphasized as a consequence of the top-illumination, and as illumination power increases the signals from the nucleus saturates the image before the membrane sieve plates can be imaged. The images were acquired using a 50X 0.8NA objective lens. The $532 \mathrm{~nm}$ excitation laser was operated in the range of 200-300 $\mathrm{mW}$ for waveguide imaging, with exposure time of $300 \mathrm{~ms}$. The fairly high power is needed due to high losses associated with the coupling loss and the propagation loss. The estimated guided power inside the waveguide is less than $5 \mathrm{~mW}$. For epi-illumination considerably less power is needed to achieve the appropriate illumination of the fluoreophores (in the range of 10-20 mW). In separate experiments, human LSECs were stained with Alexa 647 Phalloidin and imaged using waveguide excitation (laser $638 \mathrm{~nm}$ ). The images of actin in human LSEC are shown in Figure 5. The images were acquired by a water immersion objective lens (60X 1.2NA). The actin filaments are used to measure the resolution of the method, and found to be $295 \mathrm{~nm}$ at full width 
half maximum (FWHM) after a deconvolution algorithm is employed on the acquired image. Due to rapid decay of the evanescent field from the waveguide surface, low background noise is obtained in chip-based microscopy.

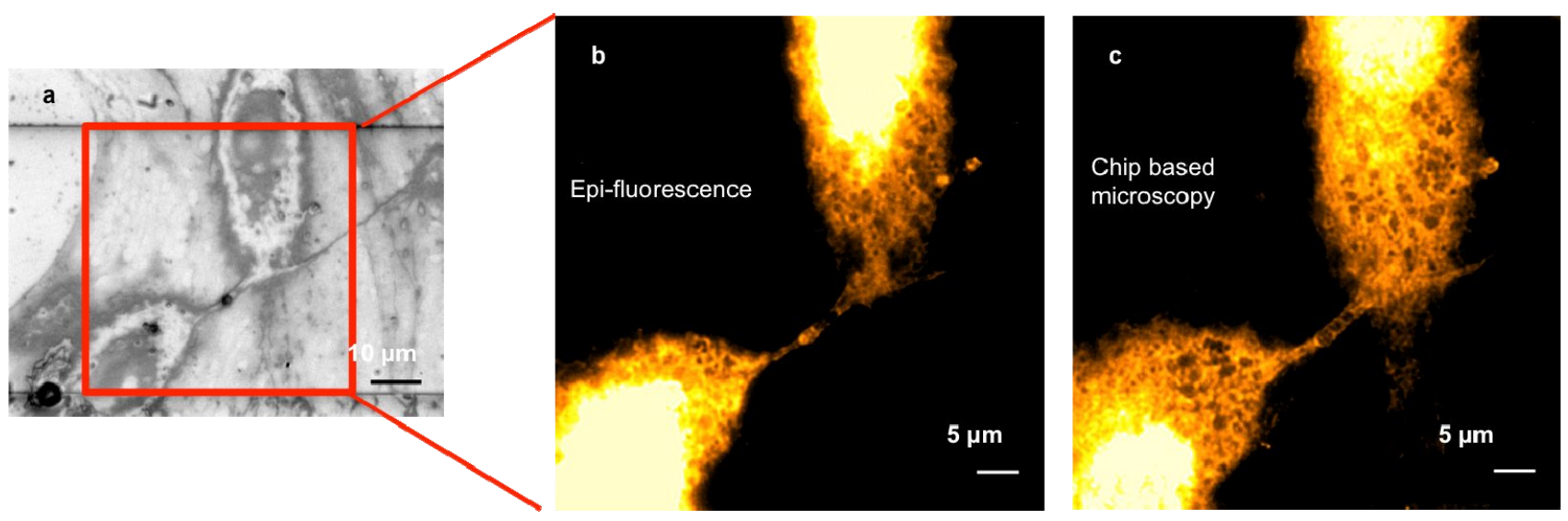

Figure 4: a) Bright field image of rat LSECs located on top of the waveguide surface. b) Waveguide TIRF image of cell region. The sieve plates present on cell membrane are imaged (dark areas present on cell membrane),c) Epi-fluorescence image of same cells.
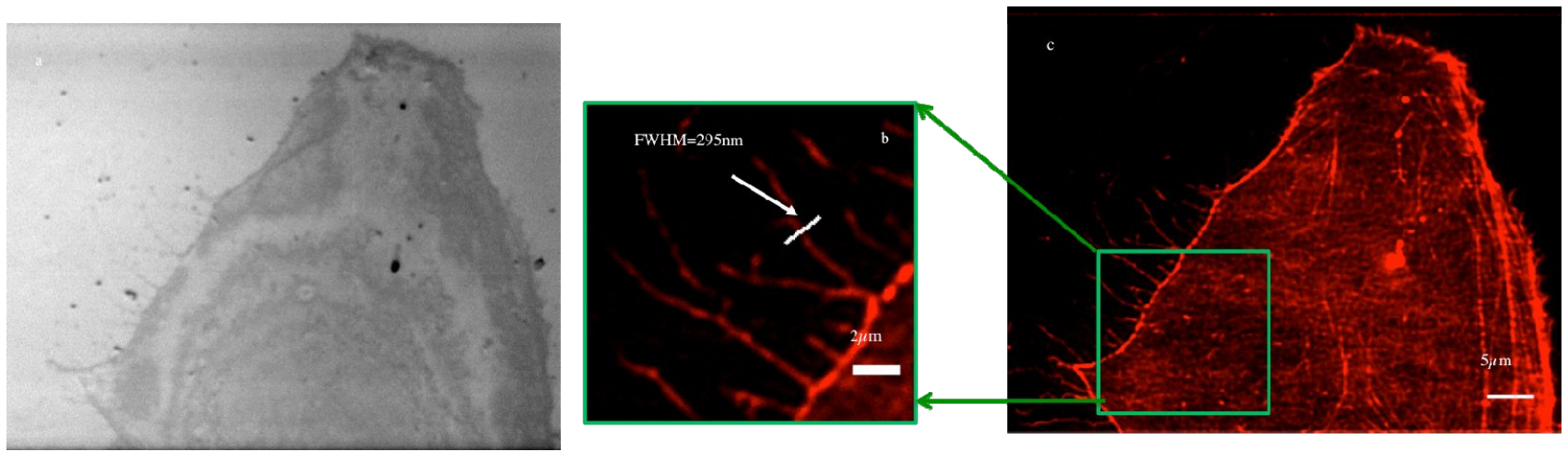

Figure 5: a) Bright field image of human LSEC on top of waveguide. b-c) Waveguide TRIF image of the same cell. The cell is stained with Alexa 647-phalloidin exposing cell actin. The FWHM is $295 \mathrm{~nm}$.

\section{CONCLUSION}

We have used waveguide-chips made of high-refractive index contrast material $\left(\mathrm{Ta}_{2} \mathrm{O}_{5}\right)$ for imaging membrane sieve plates and actins present in rat and human LSECs, respectively. In waveguide excitation, the evanescent field is dominant only near the surface $(\sim 100-150 \mathrm{~nm})$ providing default optical sectioning and illuminating fluorophores that are in close proximity to the surface and thus benefiting higher signal-to-noise ratio. Optical waveguides provide a uniform excitation over large sample area and the method represents an integrated, on-chip approach for fluorescent imaging, with a possible extension towards super-resolution imaging methods. Waveguide-chip generates evanescent field along the entire length of the waveguide, when combined with low magnification, a large field-of-view can be imaged under TIR illumination. While, in conventional TIRF microscopy both imaging and excitation are coupled thus the field-of-view is limited by TIR lens (usually high magnification). For fluorescence microscopy, it is an advantage to stain different parts of a cell with different dyes and excite each dye with a different wavelength. The integrated chipplatform is compatible with optical fiber components which would allow easy multiplexing of different wavelengths for multi-color imaging. Combining waveguide imaging with planar waveguide trapping opens for the possibility of trapping particles (cells, bacteria etc.) on the waveguide, while aquiring TIRF images of the particles [12]. 


\section{ACKNOWLEDGEMENTS}

This work was supported by the European Union, European Research Council (ERC) Starting Grant "Nanoscopy" (No. 336716) and the FP7-HEALTH-2010-Alternative-Testing "Hepatic Microfluidic Bioreactor" (AOR 266777). The authors acknowledge valuable assistance from Olav G Helleso, Deanna L.Wolfson and Jean C Tinguely.

\section{REFERENCES}

[1] H. Schneckenburger, "Total internal reflection fluorescence microscopy: technical innovations and novel applications", Current Opinion in Biotechnology 16: 13-18 (2005).

[2] Beck, M., Aschwanden, M., Stemmer, A. "Sub-100-nanometre resolution in total internal reflection fluorescence microscopy", Journal of Microscopy 232: 99-105 (2008).

[3] B. Agnarsson, S. Ingthorsson, T. Gudjonsson, and K. Leosson, "Evanescent-wave fluorescence microscopy using symmetric planar waveguides," Opt. Express 17(7), 5075-5082 (2009).

[4] B. Agnarsson, J. Halldorsson, N. Arnfinnsdottir, S. Ingthorsson, T. Gudjonsson, and K. Leosson, "Fabrication of planar polymer waveguides for evanescent-wave sensing in aqueous environments," Microelectron. Eng. 87(1), 56-61 (2010).

[5] B. Agnarsson, A.B. Jonsdottir, N.B. Arnfinnsdottir, K. Leosson, On-chip modulation of evanescent illumination and live-cell imaging with polymer waveguides, Optics Express 19, 22929-22935 (2011)

[6] Cogger V, McNerney G, Nyunt T, DeLeve L, McCourt P, Smedsrød B, Le Couteur D, Huser TR "Three-dimensional structured illumination microscopy of liver sinusoidal endothelial cell fenestrations”, Journal of Structural Biology 171:382388 (2010).

[7] Braet F, "How molecular microscopy revealed new insights in the dynamics of hepatic endothelial fenestrae in the past decade", Liver Int 24:532-539, (2004).

[8] R. Fraser, V.C. Cogger, B. Dobbs, H. Jamieson, A. Warren, S.N. Hilmer, and D.G. Le Couteur, "The liver sieve and atherosclerosis", Pathology 44:181-186, (2012)

[9] D.G. Le Couteur, R. Fraser, S.N. Hilmer, L.P. Rivory and A.J. McLean, "The hepatic sinusoid in aging and cirrhosis: effects on hepatic substrate deposition and drug clearance", Clin Pharmacokinet 44:187-200 (2005)

[10] Ahluwalia, B., S, Subramanian, A., Z., Hellesø, O., G., Perney, N., M., B., Sessions, N., P., Wilkinson, J., S., "Fabrication of sub-micron high refractive index tantalum pentoxide waveguides for optical propulsion of microparticles," Photon. Tech. Lett. 21, 1408-1410 (2009).

[11] B. P. S. Ahluwalia, Olav Gaute Hellesø; Ananth Z. Subramanian; James S. Wilkinson; Jie Chen; Xuyuan Chen., "Integrated platform based on high refractive index contrast waveguide for optical guiding and sorting", Proc. SPIE 7613, 76130R (2010).

[12] Øystein, Ivar Helle, Balpreet Singh Ahluwalia, Olav Gaute, Hellesø, "Optical transport, lifting and trapping of microparticles by planar waveguides", Opt Exp., 23(5), (2015).

[13] Balpreet S Ahluwalia, Peter McCourt, Ana Oteiza, James S. Wilkinson, Thomas R. Huser and Olav G Hellesø, "Squeezing red blood cells with the evanescent field of an optical waveguide to monitor cell deformability during blood storage", Analyst, 140, 223-229, (2015).

[14] B. S. Ahluwalia, Peter McCourt, Thomas Huser and Olav Gaute Hellesø, "Optical Trapping and propulsion of red blood cells on waveguide surfaces", Optics Express, 18, 21053 (2010).

[15] Hellesø, O., G., Løvhaugen, P., Subramanian, A., Z., Wilkinson, J., S., Ahluwalia, B., S., "Surface transport and stable trapping of particles and cells by an optical waveguide loop," Lab-on-a-chip, 12(18), 3436-40 (2012).

[16] Smedsrod, B. and H. Pertoft, "Preparation of pure hepatocytes and reticuloendothelial cells in high yield from a single rat liver by means of Percoll centrifugation and selective adherence", J Leukoc. Biol, 38(2), 213-30, (1985). 